\title{
Physician Well-Being: Expanding the Triple Aim
}

\author{
Colin P. West, MD, PhD ${ }^{1,2}$ \\ 'Division of General Internal Medicine, Department of Medicine, Mayo Clinic, Rochester, MN, USA; ${ }^{2}$ Division of Biomedical Statistics and Informatics, \\ Department of Health Sciences Research, Mayo Clinic, Rochester, MN, USA.
}

KEY WORDS: well-being; physician; healthcare system; quality improvement; burnout.

$\mathrm{J}$ Gen Intern Med 31(5):458-9

DOI: $10.1007 / \mathrm{s} 11606-016-3641-2$

(c) Society of General Internal Medicine 2016

$\mathrm{P}$ hysician well-being has become an increasing concern in recent years, reflecting mounting evidence of an epidemic of burnout and stress among medical professionals with serious attendant consequences for patients, physicians, and the medical profession. ${ }^{1}$ Roughly half of physicians in the United States suffer from burnout, and every specialty of medicine is affected. ${ }^{1}$ As such, this issue impacts hundreds of thousands of physicians and millions of patients each year. Literature on solutions to this problem remains limited, but points to the necessity for approaches directed at both individual physicians and the larger organizational environments in which they work. $^{2}$

Despite the growing understanding of the importance of promoting physician well-being, generating institutional or profession-wide standards around well-being has proved challenging. Physician well-being has been recommended as an indicator of healthcare quality given its association with patient safety and outcomes. ${ }^{2}$ A natural result of this framing would be a need for widespread measurement of physician well-being, with appropriate response to suboptimal institutional performance. Metrics for the physician experience should be embedded within institutional performance "dashboards" alongside more traditional financial metrics. Indeed, this approach appears to be taking hold across a growing number of enlightened healthcare organizations.

More recently, it has been recommended that physician well-being be added to the Triple Aim of improving the patient experience of care (including quality and satisfaction), improving the health of populations, and reducing the per capita cost of healthcare, thus creating a new Quadruple Aim., ${ }^{3,4}$ The Triple Aim is critically important to advancing medical care across the globe. However, considering healthcare innovations without direct concern for the well-being of the individuals providing care seems at best a missed opportunity for

Received November 5, 2015

Revised January 5, 2016

Accepted February 12, 2016

Published online February 26, 2016 engagement of physicians to ensure the success of these innovations and at worst a denigration of the physician role in healthcare delivery as a member of the medical profession.

Although this comment focuses on physicians' well-being because the evidence on prevalence and consequences of threats to well-being is arguably strongest for this group, the principles espoused herein apply equally well to nonphysician health professionals. The suggestions made in this paper should be carefully considered for application beyond physicians, and the impact of including all healthcare professionals in a truly inclusive Quadruple Aim addressing the entire healthcare system would be transformative.

One might assume that efforts to promote the Triple Aim should account for all factors affecting these goals, including physician well-being. However, the current state of physician well-being necessitates a more visible emphasis on this crucial contributor to the Triple Aim. The new Quadruple Aim would elevate the importance of clinician well-being to a position paralleling the original Triple Aim goals in considering healthcare-related initiatives. This aligns well with earlier calls to incorporate physician well-being into discussions of institutional performance in healthcare delivery, ${ }^{2}$ and has the potential to save us from ill-conceived approaches that may advance a Triple Aim goal but are, in fact, counterproductive because of adverse effects on physician well-being and work satisfaction. ${ }^{3,4}$ In short, changes to healthcare delivery which do not consider their impact on clinician well-being are unlikely to succeed in the short term and may have significant detrimental effects on healthcare in the long term.

How might the Quadruple Aim be implemented? First, physician well-being should be recognized as a central element of professionalism in medicine, and therefore as a professional responsibility shared by both individual physicians and the healthcare systems in which they impact patients. A focus on physician well-being should not be viewed as selfish or self-serving. Rather, physician well-being is necessary for optimal healthcare delivery in the present and a sustainable healthcare system in the future, and this necessity should be formalized in our professional standards. Without this common understanding and reinforcement of the importance of physician well-being, strategies targeting aspects of the Quadruple Aim while negatively affecting physician well-being are too readily rationalized or even prioritized.

With this integration of clinician well-being into the professional fabric of medicine, measurement of organizational 
performance in meeting this standard shifts from a novel concept to an expected event. Well-validated metrics are widely available for this purpose, and can address multiple aspects of the physician well-being spectrum, from burnout and stress to resilience and engagement. ${ }^{2}$ Importantly, although at the individual level a menu of resources to promote well-being is needed, these metrics would need to guide institutional responses and must not be used to target or further stigmatize individual physicians. A problem such as burnout known to affect fully half of all physicians is clearly an issue that is more system-driven than individual-driven, and the primary responsibility for improvement rests at the system level.

Successful implementation of the Quadruple Aim also requires an expanded knowledge of how best to promote clinician well-being. Under the Quadruple Aim, quality improvement efforts and other rigorous research on interventions to reduce distress and improve well-being become priorities considered on an equal plane with all other efforts to improve healthcare delivery. Merging consideration of physician well-being with current healthcare delivery research efforts will more rapidly and successfully lead to new knowledge on healthcare system improvements which are sustainable because they benefit all members of the system. In fact, evidence supports the association of greater clinician satisfaction with enhanced organizational performance, with a clearly positive return on investment for efforts to promote physician satisfaction. ${ }^{5}$

Integration of the Quadruple Aim into current healthcare initiatives may be aided by consideration of five key drivers of physician satisfaction: i) work effort, ii) work efficiency and support, iii) management of work-home interference, iv) flexibility and control at work, and v) values and meaning in work. These drivers have been well characterized in the literature, ${ }^{6}$ and offer a template for areas on which organizations might focus attention. Two brief examples will illustrate this point. First, increasing physician effort while reducing payment for services might be expected to reduce the per capita cost of healthcare, but may also increase physician work hours and worsen work-home interference, thereby decreasing physician satisfaction and increasing burnout and stress. This negative effect could then impede the Triple Aim goal of improving the patient experience of care. Conversely, unchecked medical fees and restricted physician work hours might improve physician well-being but limit access to care and lead to unsustainable cost increases. By forcing these sets of seemingly contradictory effects into the same discussion, we can develop a more comprehensive understanding of the expected impact of proposed changes and make better decisions for the entire healthcare system.

Second, electronic health record advances such as embedded decision support services, if integrated effectively into physicians' practices, have the potential to improve the health of individuals and populations. However, if these advances serve to deflect physician effort into nonessential and indirect activities over which they feel no control, this improved technology may actually reduce work efficiency and physicians' sense of autonomy, thereby adversely affecting physician well-being. Furthermore, innovations which pull physicians away from their patients or interfere with the physician-patient relationship may threaten the most meaningful aspects of physicians' work by dehumanizing healthcare interactions. On the other hand, lack of standardization in medical care contributes to inequities and lower-quality healthcare delivery, so there must be a balance between physician autonomy and the needs of patients. Again, the Quadruple Aim would ensure that changes to the healthcare system optimally serve the entire system, including individual patients, populations, and the professionals engaged in delivering care.

In summary, the Triple Aim of improving the patient experience of care, improving the health of populations, and reducing the per capita cost of healthcare will benefit from expansion to a Quadruple Aim that includes the goal of improving the well-being of health professionals. This addition would not detract from the Triple Aim, but rather would result in improvements to the healthcare system that effectively balance the impacts on patients, populations, and clinicians. In this way, healthcare initiatives could be considered from a deeper perspective that benefits both patients and clinicians, and in so doing maximizes delivery of quality outcomes.

Corresponding Author: Colin P. West, MD, PhD; Division of General Internal Medicine, Department of Medicine, Mayo Clinic, 200 First Street SW, Rochester, MN 55905, USA (e-mail: west.colin@mayo.edu).

\section{Compliance with Ethical Standards:}

Conflict of Interest: The author declares that he does not have a conflict of interest.

\section{REFERENCES}

1. Shanafelt TD, Boone S, Tan L, et al. Burnout and satisfaction with worklife balance among US physicians relative to the general US population. Arch Intern Med. 2012;172:1377-85.

2. Wallace JE, Lemaire JB, Ghali WA. Physician wellness: a missing quality indicator. Lancet. 2009;374:1714-21.

3. Bodenheimer T, Sinsky C. From Triple to Quadruple Aim: care of the patient requires care of the provider. Ann Fam Med. 2014;12:573-6.

4. Sikka R, Morath JM, Leape L. The Quadruple Aim: care, health, cost and meaning in work. BMJ Qual Saf. 2015;24:608-10.

5. Reid RJ, Coleman K, Johnson EA, et al. The Group Health medical home at year two: cost savings, higher patient satisfaction, and less burnout for providers. Health Aff. 2010;29:835-43.

6. Friedberg MW, Chen PG, Van Busum KR, et al. Factors affecting physician professional satisfaction and their implications for patient care, health systems, and health policy. Santa Monica, CA: RAND Corporation; 2013. Available at: http://www.rand.org/pubs/research_reports/RR439.html. Accessed January 4, 2016. 